\title{
The effect of visual biofeedback on balance in elderly population: a systematic review
}

\author{
This article was published in the following Dove Press journal: \\ Clinical Interventions in Aging \\ 6 March 2017 \\ Number of times this article has been viewed
}

\author{
Hammad Alhasan' \\ Victoria Hood ${ }^{2}$ \\ Frederick Mainwaring ${ }^{2}$ \\ 'Physiotherapy Department, Faculty \\ of Applied Medical Science, Umm \\ al-Qura University, Mecca, Kingdom \\ of Saudi Arabia; ${ }^{2}$ School of Health \\ Science, University of Nottingham, \\ Nottingham, Nottinghamshire, UK
}

Correspondence: Hammad Alhasan PO Box 9195, Alrabiah District, Mecca 21955, Makkah, Saudi Arabia

Tel +9665555I 6226

Email hshasan@uqu.edu.sa
Background: Balance is commonly affected by multiple factors, especially among the elderly population. Visual biofeedback (VBF) is an intervention tool that can be used in balance rehabilitation.

Aim: This study aimed to systematically review randomized controlled trials that examine whether VBF training is effective in improving balance in an elderly population.

Data sources: Three databases were searched: CIAHL, EMBASE, and MEDLINE. The searches were limited to the period from 2010 to 2016.

Eligibility criteria: Healthy adults, aged $\geq 65$ years, with no specific disorders were included. Interventions were any VBF intervention with the aim of improving balance and were compared to no intervention, traditional exercises, placebo, or standard care. The outcome measures were balance as measured by any validated outcome measure.

Studies appraisal method: The Physiotherapy Evidence Database quality assessment tool and The Cochrane Collaboration tool for assessing risk of bias were used by two independent authors (HA and FM) in order to appraise the included studies.

Results: The database search resulted in 879 articles, of which five papers were included. VBF was compared to no intervention, a placebo, and traditional exercise. The total number of participants in all the five included studies was 181 , with a mean age of 74.3 years (standard deviation 6.7). Two studies were rated as high-quality studies, and three were rated as fair quality.

Conclusion: Engaging elderly people living in the community in VBF training was found to be effective and could improve their balance ability. However, the variation between studies in methodology, intervention protocol, and outcomes utilized made it difficult to inform a definitive statement regarding the potential application of VBF for balance training with the elderly. Furthermore, high-quality randomized control trials are required. The systematic review level of evidence is moderate, and the strength of recommendation is that VBF is likely to be beneficial.

Keywords: visual feedback, exergames, older adult, postural balance

\section{Introduction}

Balance impairments in older adults are one of the most experienced and reported problems to physicians, ${ }^{1}$ and balance and gait disorders are the second leading causes of falls. ${ }^{2}$ Literature has suggested that between $20 \%$ and $33 \%$ of adults aged $>65$ years experience problems with their balance. ${ }^{3}$ In adults aged $>60$ years, there is a $30 \%$ chance of falling at least one time a year, and this chance increases up to $45 \%$ in those aged $\geq 70$ years. ${ }^{4}$ It is estimated that falls cost the United Kingdom's National Health Service approximately $£ 2.3$ billion every year. ${ }^{5}$

The human balance system is a complicated one, which involves integration and coordination of sensory, motor, and biomechanical activities in such a way as 
to halt falls and enhance static and dynamic performance. The failure of coordination in one of these activities has the potential to result in an abnormal movement of the human body, which could be seen as a sway, a loss of balance, or a possible fall. ${ }^{6}$

The effect of exercise on reducing falls and improved balance are well documented and a primary component in public guidelines for fall prevention. ${ }^{5}$ Biofeedback intervention provides individuals with additional information about their body function with the purpose of developing changes in behavior that lead to better and enhanced performance. ${ }^{7}$ Biofeedback can be considered as a training method rather than a treatment. When individuals undergo biofeedback training, they should be active learners and practice until the skill of controlling body motion is developed. A majority of biofeedback systems, especially wearable devices described in the literature, have been designed to be used in laboratories and clinics. ${ }^{8}$ To help with training in the community, an alternative and inexpensive device is preferable. Because of its ease of access and cost-effectiveness, game consoles, such as the Nintendo ${ }^{\circledR}$ Wii Fit, have become popular a training tool.

Nintendo Wii is one of the most popular devices being used for rehabilitation with the elderly. It consists of a Wii balance board (WBB) with a platform to monitor any change in an individual's center of pressure ( $\mathrm{CoP})$ and an innovative remote with a three-dimensional accelerometer technology to track body movement. The board and the remote are connected to the Wii Fit software that provides feedback about any change in CoP through auditory and visual feedback. ${ }^{9}$

Seven randomized control trials (RCTs) were included in a systematic review conducted by Laufer et al, ${ }^{10}$ regarding the effect of Nintendo Wii exercises on balance in healthy, elderly population; ${ }^{10}$ the majority were considered to be of fair quality on the Physiotherapy Evidence Database (PEDro) scale with a total number of 285 participants. Outcome measures were any valid functional balance measure, and in general, the results showed better outcomes with the use of Wii Fit against traditional exercises; however, no firm decision on the effectiveness of the Wii Fit was reported because of great variability between the studies in terms of protocols and outcome measurements.

However, Rodrigues et $\mathrm{al}^{11}$ contradicted the results of Laufer et al. ${ }^{10}$ Rodrigues et al aimed to evaluate the effect of exergaming on musculoskeletal function among the elderly. An important point is that they included both healthy and unhealthy people in their sample of participants. Sixteen studies of different designs were included with a total of
532 elderly participants, and functional balance assessment was one of the outcome measures, which was used as an inclusion criterion. The review concludes that there is no evidence that exergaming may be effective on musculoskeletal function and balance. Unfortunately, more than half of the included studies were of low quality; some studies had methodological errors, for example, they did not mention the type of games used or the duration, there was no standardization of the assessment and training which resulted in significant heterogeneity in the meta-analysis, and no blinding was reported in any of the included studies.

The current systematic review (SR) is an update of the previous work by Agnes et $\mathrm{al}^{12}$ in which they evaluated the effect and feasibility of biofeedback on balance among the elderly. Four studies were included, and the number of participants ranged from 5 to 30 . However, the researchers were unable to conclude because of the variation in the studies, including the small sample sizes.

According to the systematic reviews mentioned earlier, it is evident that the use of exergaming and virtual reality is safe and feasible for most participants. Nevertheless, the effectiveness of virtual reality and exergaming is inconclusive. There is an unclear evidence with regard to the use of visual biofeedback (VBF) as an intervention for balance problems, leading to the conclusion that a greater number of larger RCTs are needed. In the last few years, several RCTs have been published; therefore, the aim of this review was to summarize the more recent evidence to propose a decision with regard to the effectiveness of VBF on balance among the healthy elderly.

\section{Materials and methods Data sources}

This review is an update of the review conducted by Agnes et al, ${ }^{12}$ in which electronic databases were searched from 1990 to 2010. The current search was conducted from January 2010 to June 2016 to extract recent evidence about the effect of VBF on balance in healthy, older people. These databases were: CIAHL, EMBASE, and MEDLINE. After all the databases were searched, duplicate publications were removed. Two authors (HA and FM) independently conducted the search; the final search was conducted in June 2016.

\section{Search strategies}

Each database was searched with a different search strategy tailored to the search terms specific to that database. Table 1 describes the strategy used for the CINHAL 
Table I CINHAL database search strategy

\begin{tabular}{|c|c|}
\hline & Search terms \\
\hline \multirow[t]{2}{*}{ SI } & (MH “Biofeedback”) OR “biofeedback" OR “bio-feedback” OR “augmented feedback” OR “visual feedback" OR (MH “Video Games”) OR \\
\hline & "video Games” OR “computer games” OR “virtual reality” \\
\hline \multirow[t]{2}{*}{ S2 } & (MH “Balance, Postural”) OR “postural balance” OR “equilibrium” OR “balance” OR (MH “Posture”) OR “posture” OR “Musculoskeletal \\
\hline & Equilibrium" \\
\hline S3 & (MH “Aged") OR "aged" OR “older people" OR “old people" OR “older persons" OR “older subjects" OR “old persons" OR “old subjects" \\
\hline S4 & SI AND S2 AND S3 \\
\hline
\end{tabular}

database. Table S1 summarizes the search strategy used for the MEDLINE and EMBASE databases.

\section{Study selection}

All parallel RCTs published between 2010 and 2016 in which a VBF intervention was compared to no intervention or other interventions with the aim of improving balance in elderly people were included. Study designs other than those mentioned previously were excluded. Table 2 describes the PICO (Patient, Intervention, Comparison, Outcome) used for this review.

\section{Summary of participants}

All subjects, male or female, described as older adults, geriatric, aged or elderly, with a mean age $\geq 65$ years, living in the community or independently in retirement centers, were included. Studies that included participants with specific medical conditions, such as stroke, Parkinson's disease, cognitive impairment, diabetes, fracture, amputation, osteoarthritis, psychiatric, cardiac, neurological, and other specific medical conditions were excluded. Participants described as frail were also excluded, as this term may be used in the literature to describe people with different conditions, including general debility and cognitive impairment.

\section{Type of intervention}

RCTs that used any form of VBF, including exergames, aimed directly at improving balance, which fall into the category of devices that use inertial sensors, and other devices that use camera system technology were included. Specifically, trials that compare the following were included:

Table 2 PICO for the review

\begin{tabular}{ll}
\hline P: Population & $\begin{array}{l}\text { Healthy adults aged } \geq 65 \text { years, with no specific } \\
\text { disorders or health conditions }\end{array}$ \\
I: Intervention & $\begin{array}{l}\text { VBF (visual, video games, virtual reality) with the aim } \\
\text { of improving balance }\end{array}$ \\
C: Comparison & $\begin{array}{l}\text { No intervention, traditional exercises, placebo, or } \\
\text { standard care }\end{array}$ \\
O: Outcomes & $\begin{array}{l}\text { Balance as measured by any validated outcome } \\
\text { measure }\end{array}$
\end{tabular}

Abbreviation: VBF, visual biofeedback.
- VBF compared to traditional therapy or standard care.

- VBF compared to other balance interventions without VBF.

- VBF compared to control or placebo or no treatment.

\section{Type of outcome measure}

Studies that used any validated standardized outcome measure designed to measure static and dynamic balance in elderly people, such as the Berg Balance Scale (BBS), ${ }^{13}$ Timed Up and Go (TUG), ${ }^{14}$ and objective balance measures by force platform systems, were included.

\section{Quality assessment}

The PEDro quality assessment tool was used by two authors (HA and FM) to appraise the included studies. According to Herd et al, ${ }^{15}$ the total score obtained from PEDro indicates the strength of the study:

- If the total score was $\geq 6$, it is considered a high-quality study.

- If the total score was between 4 and 5, it is considered a fair-quality study.

- If the total score was $\leq 3$, it is considered a poor-quality study.

To assess the risk of bias in the included studies, the Cochrane Collaboration tool for assessing risk of bias (CCTARB) was used. Because of the difficulty of blinding the therapist and subjects in all physiotherapy interventions, ${ }^{16}$ the blinding of personnel and participants will not be counted as a key domain, whereas the remaining domains will be considered as key domains.

\section{Data extraction}

Trials were screened by two authors (HA and FM) for eligibility by reviewing the title and abstract. If the title or the abstract was perceived to be relevant, the full text of the paper was retrieved to evaluate it against the inclusion and exclusion criteria. Any disagreement between the authors was solved by a third author ( $\mathrm{VH})$. A data extraction form was created, which included a trial setting (hospital or 
community); method (design, method of randomization, and type of visual biofeedback [VBF] used); characteristics of participants (gender, age, criteria of inclusion and exclusion, and number of participants in each group); intervention (duration and type of intervention); outcome measure used, and the result. The data were extracted independently by the two authors.

\section{Data analysis}

The effect size was calculated where possible using RevMan 5.3 for Windows. The standardized mean difference (SMD) was used as a summary effect size estimator. The Cohen's $d$ formula was used to calculate the effect size, which is a measure of the distance between two means, measured in standard deviations (SDs). ${ }^{17}$

A descriptive analysis of the included trials was conducted to evaluate the effect of VBF on balance among elderly people. PEDro scores and CCTARB were used to compare the included studies.

\section{Results}

A total of 672 papers were identified as relevant. After the initial screening of the papers, 644 were excluded based upon the titles and abstracts, the remaining 28 papers were screened after retrieving the full text, of which 23 were excluded for failing to meet the inclusion criteria, that is, not in English language or not an outcome of interest. The final review included five papers that met the inclusion/exclusion criteria. The process of selecting the studies in this systematic review is presented in Figure 1. Characteristics of the final five studies retrieved are summarized in Table 3.

\section{Methodological quality}

The appraisal of the included studies according to PEDro scale is presented in Table 4. The mean PEDro score was 5.2 (SD 1.3), with two studies ${ }^{18,19}$ graded as high quality and the remaining three ${ }^{20-22}$ graded as fair quality. Four items in the PEDro scale were positive in all studies: random allocation, baseline comparability, between-group comparisons, and point estimates and variability. In contrast, blinding of the participants or the therapists was impossible because of the nature of the studies, and only two studies maintained blinding of the assessor. ${ }^{18,19}$ Finally, concealed allocation was absent in all studies, and intention to treat was positive in only two studies. ${ }^{18,19}$

\section{Risk of bias assessment}

Table 5 summarizes the assessment of risk of bias across all the included studies. Figure 2 shows the risk of bias graph.

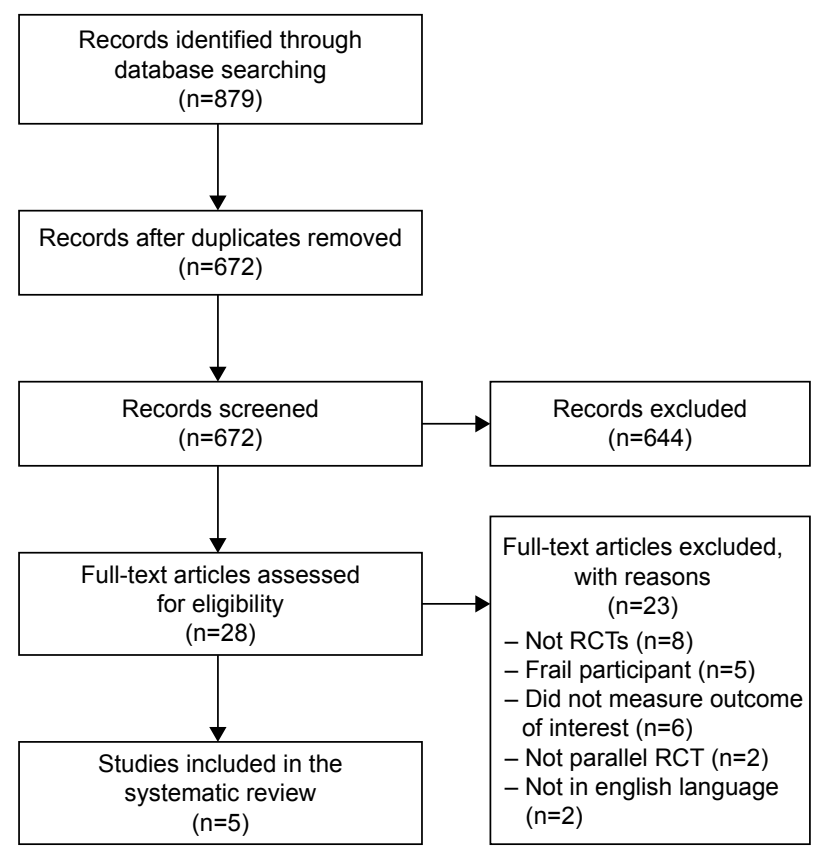

Figure I Results of the literature search conducted in June 2016. Abbreviation: RCT, randomized control trial.

\section{Random sequence generation}

One study ${ }^{19}$ had a low risk of bias as it reported random sequence generation (randomized by computer-generated random numbers). Two studies ${ }^{18,21}$ had an unclear risk of bias as they reported that participants were randomized with no information of the procedure of randomization. Two studies $^{20,22}$ had a high risk of bias as they did not report that both groups were randomized.

\section{Allocation concealment}

One study ${ }^{19}$ had a low risk of bias as the concealment procedures were stated clearly. All the four ${ }^{18,20-22}$ remaining studies had a high risk of bias as there was no allocation concealment reported by any of the studies.

\section{Blinding of participants and personnel}

All the five studies had an unclear risk of bias as they did not report any information regarding this. However, as mentioned earlier, this domain is not considered as one of the key domains.

\section{Blinding of outcome assessment}

Two studies ${ }^{18,19}$ had a low risk of bias. The remaining three ${ }^{20-22}$ studies had unclear risk of bias as there was insufficient information to make a judgment.

\section{Incomplete outcome data}

All the studies had a low risk of bias as no missing data were reported. 


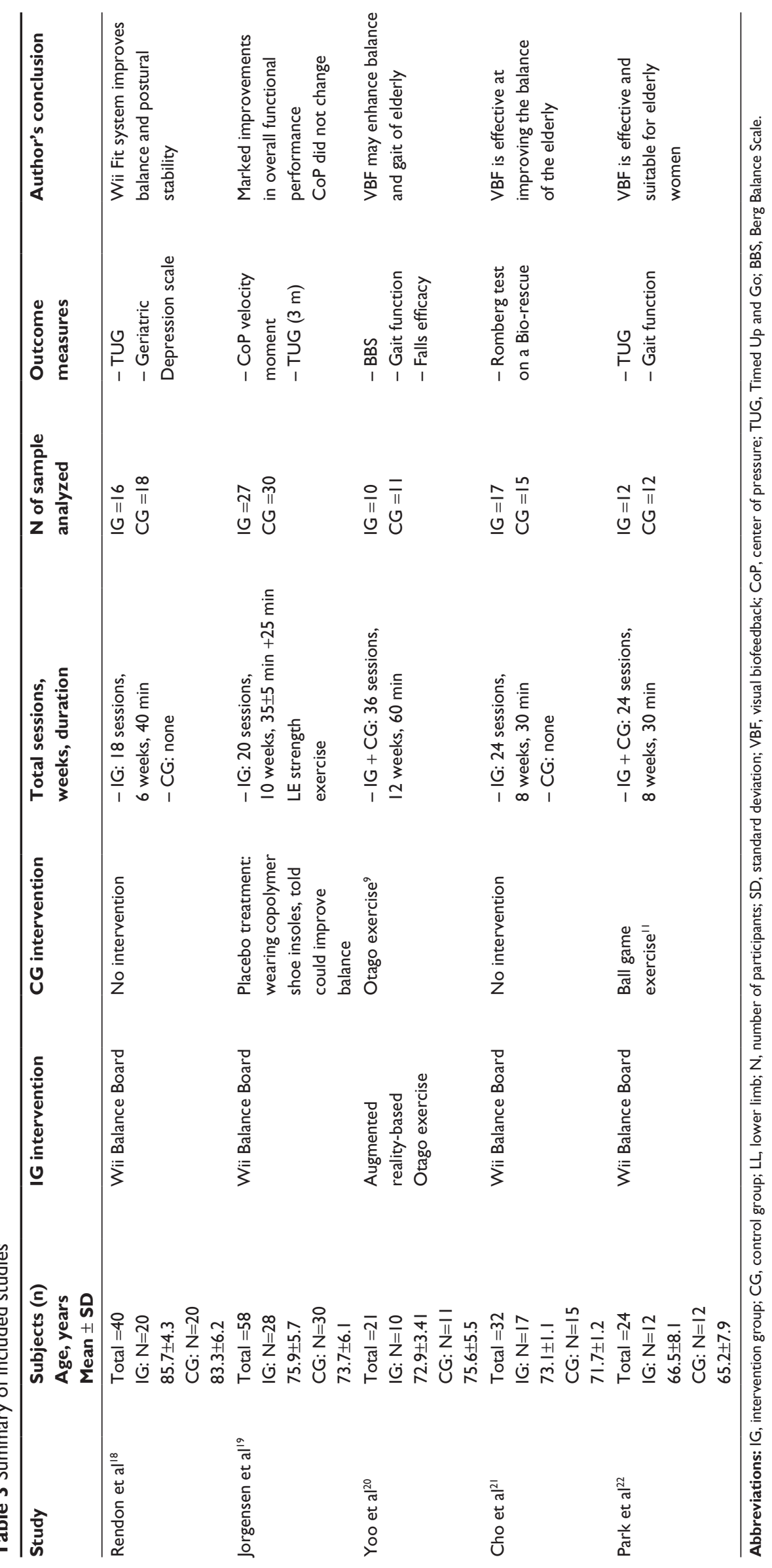


Table 4 PEDro score for the included studies

\begin{tabular}{|c|c|c|c|c|c|c|c|c|c|c|c|c|c|}
\hline Study & Year & 1 & 2 & 3 & 4 & 5 & 6 & 7 & 8 & 9 & 10 & II & Total \\
\hline Rendon et al ${ }^{18}$ & 2012 & $\mathrm{~N}$ & $Y$ & $\mathrm{~N}$ & $Y$ & $\mathrm{~N}$ & $\mathrm{~N}$ & $Y$ & $\mathrm{~N}$ & $Y$ & $Y$ & $Y$ & $6 / 10$ \\
\hline Jorgensen et al ${ }^{19}$ & 2013 & $Y$ & $Y$ & $\mathrm{~N}$ & $Y$ & $\mathrm{~N}$ & $\mathrm{~N}$ & $Y$ & $Y$ & $Y$ & $Y$ & $Y$ & $7 / 10$ \\
\hline Yoo et $\mathrm{a}^{20}$ & 2013 & $\mathrm{~N}$ & Y & $\mathrm{N}$ & $Y$ & $\mathrm{~N}$ & $\mathrm{~N}$ & $\mathrm{~N}$ & $Y$ & $\mathrm{~N}$ & Y & $Y$ & $5 / 10$ \\
\hline Cho et $\mathrm{a}^{21}$ & 2014 & $\mathrm{Y}$ & $Y$ & $N$ & $Y$ & $\mathrm{~N}$ & $\mathrm{~N}$ & $\mathrm{~N}$ & $\mathrm{~N}$ & $N$ & $Y$ & $Y$ & $4 / 10$ \\
\hline Park et $\mathrm{a}^{22}$ & 2015 & $\mathrm{~N}$ & $Y$ & $\mathrm{~N}$ & $Y$ & $\mathrm{~N}$ & $\mathrm{~N}$ & $\mathrm{~N}$ & $\mathrm{~N}$ & $\mathrm{~N}$ & $Y$ & $Y$ & $4 / 10$ \\
\hline Total & & $2 / 5$ & $5 / 5$ & $0 / 5$ & $5 / 5$ & $0 / 5$ & $0 / 5$ & $2 / 5$ & $2 / 5$ & $2 / 5$ & $5 / 5$ & $5 / 5$ & \\
\hline
\end{tabular}

Abbreviations: PEDro, Physiotherapy Evidence Database; Y, yes; N, no; I, eligibility criteria; 2, random allocation; 3, concealed allocation; 4, baseline comparability; 5, blind subjects; 6, blind therapists; 7, blind assessors; 8, adequate follow-up; 9, intention-to-treat analysis; I0, between-group comparisons; II, point estimates and variability.

\section{Selective reporting}

Two studies ${ }^{18,19}$ had a low risk of bias as they followed a pre-specified protocol. The remaining three studies ${ }^{20-22}$ had an unclear risk of bias as there was insufficient information to make a judgment.

\section{Other bias}

Two studies ${ }^{18,20}$ had a low risk of bias, whereas the remaining three studies ${ }^{19,21,22}$ had an unclear risk of bias.

\section{Characteristics of included studies Subjects}

The total number of participants in all five included studies was 181 , with a mean age of 74.3 years (SD 6.7); 83 were included in the VBF group, whereas 86 were placed in the control group. In the VBF group, the number of subjects per group ranged from 10 to 28 with a mean of 16.6 (SD 6.9), whereas in the control group, the number of subjects ranged from 11 to 30 with a mean of 17.2 (SD 7.6).

Three studies were conducted in the Republic of Korea, ${ }^{20-22}$ the remaining two were in Denmark, ${ }^{19}$ and the US. ${ }^{20}$ All of the included studies were carried out in clinical settings, which were mainly hospitals.

\section{VBF intervention}

Four of the included studies used the Nintendo Wii Fit device as a training method, ${ }^{18,19,21,22}$ whereas the remaining study ${ }^{20}$ used the augmented reality-based Otago exercise in which subjects stood in front of a computer with a web camera, which had a head-mounted display and they followed the movement displayed. The computer sensed the movement of the subjects and sent the information to the head-mounted display in order to repeat the task and move to the next level, which increased the speed. ${ }^{20}$

A total of 15 games were used in all Wii studies, ${ }^{18,19,21,22}$ with the number of games per study ranging from 3 to 6 with a mean of 3.7 (SD 1.5).

In one study, ${ }^{18}$ participants were asked to wear a belt for safety, and if required, they could use additional safety precautions, such as a cane or walker. In four studies, participants practiced VBF individually, except for one study, where they practiced in pairs. ${ }^{19}$

\section{Control intervention}

Two studies ${ }^{18,21}$ received no intervention, and two ${ }^{20,22}$ received an alternative exercise program. These exercises varied between studies; one study used ball exercises, ${ }^{20}$ whereas the other study used the Otago exercise..$^{22}$ Furthermore, one study ${ }^{19}$ received a placebo treatment as they were asked to wear an ethylene vinyl acetate copolymer insoles to help improve their balance.

\section{Duration and number of treatments}

The duration of VBF sessions ranged from 6 to 12 weeks with mean of 8.8 (SD 2.2) weeks. The total number of treatments ranged from 18 to 36 with mean of 24.4 (SD 6.9) session.

Table 5 Summary of the assessment of risk of bias across all included studies

\begin{tabular}{|c|c|c|c|c|c|}
\hline Domain & Jorgensen et al ${ }^{19}$ & Yoo et $\mathrm{al}^{20}$ & Cho et $\mathrm{al}^{21}$ & Rendon et al ${ }^{18}$ & Park et $\mathrm{al}^{22}$ \\
\hline Random sequence generation & L & $\mathrm{H}$ & $?$ & $?$ & $\mathrm{H}$ \\
\hline Allocation concealment & L & $\mathrm{H}$ & $\mathrm{H}$ & $?$ & $\mathrm{H}$ \\
\hline Blinding of participants and personnel & $?$ & $?$ & $?$ & $?$ & $?$ \\
\hline Blinding of outcome assessment & L & $?$ & $?$ & L & $?$ \\
\hline Incomplete outcome data & L & L & L & L & L \\
\hline Selective reporting & L & $?$ & ? & L & $?$ \\
\hline Other bias & $?$ & L & ? & L & $?$ \\
\hline
\end{tabular}

Abbreviations: L, low risk of bias; $\mathrm{H}$, high risk of bias; ?, unclear risk of bias. 


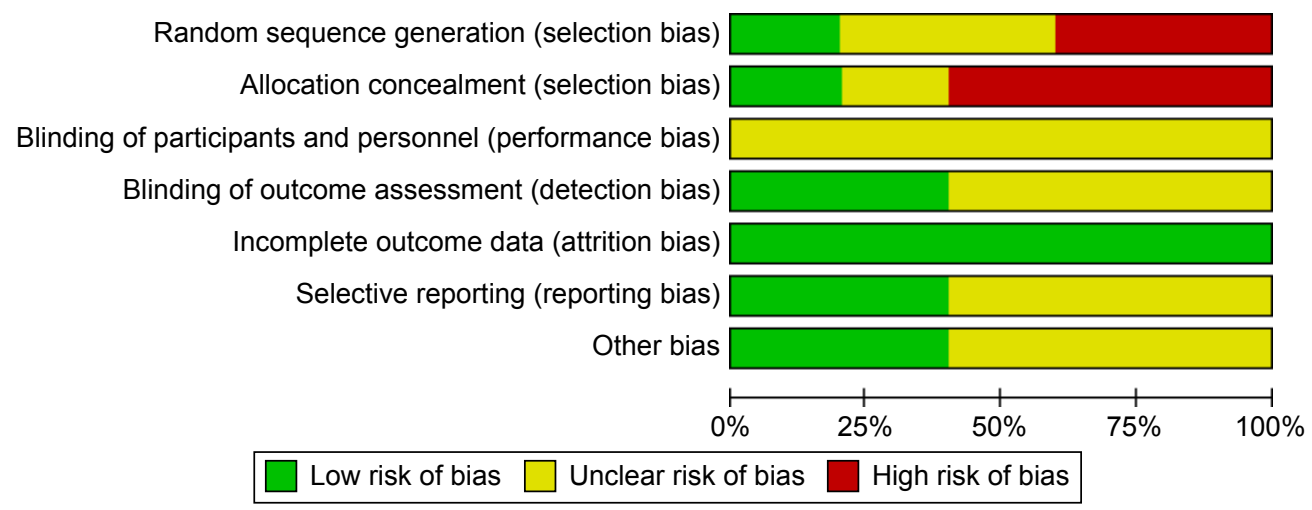

Figure 2 Review authors' judgments about each risk of bias item presented as percentages across all included studies.

The length of treatment ranged from 30 to 60 min with mean of 40 (SD 12.2) min.

\section{Outcome measures}

All studies carried out an assessment pre and post intervention. Functional balance performance was used as an outcome measure in four studies, ${ }^{18-20,22}$ three studies ${ }^{18,19,22}$ used the TUG test, and one ${ }^{20}$ used BBS. Instrumented measures of postural sway were used in three studies. ${ }^{19-21}$ One study ${ }^{19}$ used an instrumented force plate to assess postural balance capacity by analyzing the center of pressure velocity moment (CoP-VM; $\left.\mathrm{mm}^{2} / \mathrm{s}\right)$ during static bilateral stance. Two studies ${ }^{20,21}$ used an analysis biofeedback system Biorescue (RM INGENERIE, France) in which static balance was assessed with the subject's eyes open and closed while using the Romberg test.

\section{Treatment effect}

Two studies ${ }^{18,21}$ compared VBF to no intervention. Rendon et al found a significant difference between groups on TUG $(P=0.038),{ }^{18}$ and Cho et al found a significant difference between groups in body $\mathrm{CoP}$ movement area on the Romberg test with eyes closed $(P=0.001) .{ }^{21}$

In addition, two studies ${ }^{20,22}$ compared VBF against different exercise regimens, a significant difference between groups on BBS scale $(P=0.001)$ was reported by Yoo et $\mathrm{al}^{20}$ and a significant difference between groups in TUG test ( $P$-value not reported) was found in Park et al. ${ }^{22}$

One study ${ }^{19}$ compared VBF to a placebo intervention. No significant difference between groups was found in static postural balance measurement on an instrumented force plate $(P=0.92)$. However, significant difference between groups were reported in TUG $(P=0.01)$.

In addition, Park et al reported significant difference between groups on gait function in $30 \mathrm{~s}$ sway length average and sway speed. ${ }^{20}$ Three studies ${ }^{18,19,22}$ used TUG as an outcome measure, but they varied in the procedure of conducting the test. For example, one study ${ }^{18}$ used the test and had the participants walk $3 \mathrm{~m}$, in the second study ${ }^{22}$ it was $2.5 \mathrm{~m}$. Another reason for variation was that the control groups in each study varied; one study used a placebo, the other used exercise, and one used no intervention. Therefore, the effects of VBF on balance could not be pooled for a meta-analysis, because of methodological variability and the different control intervention used across studies.

\section{Effect size}

Park et $\mathrm{al}^{22}$ found that 24 sessions with 30 min of Wii training had a small effect (Cohen's $d=0.25$ ) on TUG in a group of elderly compared to those who used ball exercises. In addition, Yoo et $\mathrm{al}^{20}$ found that 36 sessions with $60 \mathrm{~min}$ of augmented reality-based Otago exercises had a small effect (Cohen's $d=0.44$ ) on BBS score in group of elderly compared to those with traditional fall exercises..$^{20}$ The same small effect (Cohen's $d=0.44$ ) was found in 20 sessions with $35 \mathrm{~min}$ of Wii training in elderly compared to a placebo intervention. ${ }^{19}$ Finally, a very large effect (Cohen's $d=1.92$ ) was found in the Romberg test with eyes closed after 24 sessions with $30 \mathrm{~min}$ of Wii training in elderly compared to no intervention. ${ }^{21}$ In the study conducted by Rendon et al, ${ }^{18}$ the effect size could not be reported because the result was reported in median and variance. Table 6 summarizes the effect size for the included studies.

\section{Discussion}

The current systematic review was conducted to evaluate the effect of VBF on balance in healthy elderly people. The literature review showed increased interest in VBF as an exercise instrument for the elderly. Five parallel RCTs evaluating the effect of VBF on balance among elderly people were 
Table 6 Summary of the effect size for the included studies

\begin{tabular}{|c|c|c|c|c|c|c|}
\hline \multirow[t]{2}{*}{ Study } & \multirow[t]{2}{*}{ Comparison } & \multirow[t]{2}{*}{ Outcome measures } & \multicolumn{2}{|c|}{$\begin{array}{l}\text { Mean difference } \\
\text { (within groups) } P \text {-value }\end{array}$} & \multirow[t]{2}{*}{$\begin{array}{l}\text { Mean difference (between } \\
\text { groups) } P \text {-value } 95 \% \mathrm{Cl}\end{array}$} & \multirow[t]{2}{*}{ Cohen's d } \\
\hline & & & CG & IG & & \\
\hline Rendon et al ${ }^{18}$ & $\begin{array}{l}\text { No } \\
\text { intervention }\end{array}$ & TUG & NR & NR & $S(P=0.038)$ & NC \\
\hline Jorgensen et al ${ }^{19}$ & $\begin{array}{l}\text { Placebo } \\
\text { intervention }\end{array}$ & $\begin{array}{l}\text { - CoP velocity } \\
\text { moment } \\
\text { - TUG }\end{array}$ & NR & NR & $\begin{array}{l}-\mathrm{NS}(P=0.92) \\
-\mathrm{S}(P=0.0 \mathrm{I})\end{array}$ & $-0.44(-0.09,0.96)$ \\
\hline Yoo et $\mathrm{al}^{20}$ & $\begin{array}{l}\text { Otago } \\
\text { exercise }^{20}\end{array}$ & BBS & $S(P=0.00 I)$ & $S(P=0.00 I)$ & $S(P=0.01)$ & $0.44(-0.43,1.3 \mathrm{I})$ \\
\hline Cho et $\mathrm{al}^{21}$ & $\begin{array}{l}\text { No } \\
\text { intervention }\end{array}$ & $\begin{array}{l}\text { Romberg test eyes } \\
\text { closed on a Bio-rescue }\end{array}$ & $S(P<0.05)$ & NS $(P>0.05)$ & $S(P=0.00 I)$ & $1.92(1.06,2.78)$ \\
\hline Park et $\mathrm{al}^{22}$ & Ball exercise ${ }^{22}$ & TUG & $\begin{array}{l}\text { NS (no } P \text {-value } \\
\text { reported) }\end{array}$ & $\begin{array}{l}S \text { (no } P \text {-value } \\
\text { reported) }\end{array}$ & $\begin{array}{l}S \text { (no } P \text {-value } \\
\text { reported) }\end{array}$ & $0.25(-0.56,1.05)$ \\
\hline
\end{tabular}

Abbreviations: CG, control group; IG, Intervention group; S, significant difference; NS, no significant difference; NR, not reported; NC, not calculated; Cl, confidence interval; CoP, center of pressure; TUG, Timed UP and Go; BBS, Berg Balance Scale.

published between 2010 and 2016. Limitation and variability in the methodology of the studies made it difficult to conduct a comprehensive meta-analysis; however, effect sizes for the majority of the studies could be determined.

All the five studies showed that VBF had a significant difference between groups. There seems to be a benefit of VBF compared to exercise and no intervention. Three studies, one ${ }^{19}$ considered to be high quality and two ${ }^{20,22}$ with moderate quality showed that there was a small effect size of VBF, whereas one moderate-quality study ${ }^{21}$ showed a very large effect size.

TUG was used in two studies, one with high quality ${ }^{19}$ and the other with moderate quality. ${ }^{22}$ Both the studies involved 82 elderly individuals and demonstrated a small effect size after VBF training compared to ball exercises $(\mathrm{SMD}=0.25$, $95 \%$ confidence interval $[\mathrm{CI}][-0.56,1.05])$ and placebo intervention $(\mathrm{SMD}=0.44,95 \%[-0.09,0.96])$. This finding is consistent with previous SR findings by Rodrigues et al, ${ }^{11}$ in which three studies (two with low quality and one high quality based upon the JADAD scale) included a total of 120 elderly individuals who were divided into two groups, a VBF group and an exercise group. TUG was used to assess balance, and a small effect was found $(\mathrm{SMD}=0.23$, $95 \%$ CI $[-0.13,0.59])$. In the review by Laufer et al, ${ }^{10}$ no effect size calculation was conducted; however, part of their findings were not consistent with the current SR as there was no significant improvement observed in the VBF group compared to the exercise group in two studies, whereas a significant improvement was found in one study only. The study that reported a significant difference was rated as high quality based upon PEDro, whereas the remaining two were of moderate quality. Overall, based upon the findings from high-quality studies, it seems that VBF has a small effect on balance as measured by TUG.

The BBS was used in the moderate-quality study by Yoo et al, ${ }^{20}$ which involved 21 elderly participants and a small effect size was found $(0.44,95 \%$ CI $[-0.43,1.31])$. This finding is consistent with the review conducted by Rodrigues et al, ${ }^{11}$ in which four studies (three with high quality and one with low quality based upon the JADAD scale) compared VBF to no intervention and found that it had a small effect (value not reported). The same result was also reported by Agnes et al. ${ }^{12}$ On the other hand, the finding from an SR by Goble et $\mathrm{al}^{23}$ contradicts this finding in which a moderatequality study compared VBF to no intervention and found that it had a large effect (Cohen's $d=0.7$ ) on BBS. The difference in the effect size could possibly be because the control in the study by Goble et $\mathrm{al}^{23}$ received no intervention, whereas the control group in the study by Yoo et $\mathrm{al}^{20}$ received ball exercises. In addition, the duration of the sessions varied between the two studies; the one that produced a small effect ${ }^{20}$ utilized $60 \mathrm{~min}$, whereas the large effect ${ }^{23}$ was produced after $15 \mathrm{~min}$ in the other study. Moreover, the studies included in Goble et $\mathrm{al}^{23}$ were not RCTs. The majority of studies, particularly those with higher quality, tend to report positive improvement but smaller effects of VBF on BBS.

With the Romberg test as an outcome, Cho et $\mathrm{al}^{21}$ found a large effect size after 30 min of Wii training (SMD $=1.92$, $95 \%$ CI $[1.06,2.78])$. This is in line with the finding by Goble et al. ${ }^{23}$ One of the studies included in their SR in which a group of elderly people received Wii training for $60 \mathrm{~min}$ compared to no intervention had a very large effect size in a Wii Fit test of center of balance (Cohen's $d=1.9$ ). This might be because the control group did not receive any intervention; meaning 
that they did not engage in any physical activity during the training period. It is possible that doing any form of exercise was better than doing nothing, as the studies that compared VBF to exercise reported lower effect sizes. An additional explanation is the small sample size in both the studies.

In the study conducted by Jorgensen et al, ${ }^{19}$ postural balance was evaluated by static bilateral testing on force platform, and no difference was found between the intervention group and placebo group. This is in contrast with Laufer et al, ${ }^{10}$ in which three studies reportedly showed a positive effect of VBF against different control groups in term of postural sway. An explanation for this is that in Jorgensen et al, ${ }^{19}$ the static bilateral test could not be challenging enough for the elderly which prevents the detection of improvement. Also the Wii uses dynamic exercises, and the use of dynamic and more challenging tests could have revealed an improvement in postural balance.

An important factor that could affect the effectiveness of any intervention is the time or duration of the training session. According to the American College of Sports Medicine Guidelines $^{24}$ (ACSM) for balance training in elderly, their recommendation is to exercise two to three times per week for 20-30 min. In this SR, only two studies followed this recommendation. The duration of VBF varies between studies with $40 \mathrm{~min}$ in Rendon et al, ${ }^{7} 35 \mathrm{~min}$ in Jorgensen et al, ${ }^{19}$ $60 \mathrm{~min}$ in Yoo et al, ${ }^{20}$ and $30 \mathrm{~min}$ in Cho et $\mathrm{al}^{21}$ and Park et al. ${ }^{22}$ There is a strong need to standardize the time and parameter for VBF intervention, as exercises with the same parameters and outcomes are necessary for comparative purposes.

The current review is able to provide a more confident summary of evidence than previous reviews. Three ${ }^{10,12,23}$ of the recent SRs did not inform a decision as to the effect of VBF, instead they report promising future for this modality. One review ${ }^{11}$ reported that there is no evidence that VBF is effective among the elderly. Overall, from the evidence, there seems to be a shift toward seeing a positive effect of VBF. More recent studies are demonstrating improvements in study design, which lead to less bias and a better ability to determine a true effect of the intervention. These higher levels of evidence show that there is a small effect of VBF. Earlier SRs could not provide a definitive decision because reviews included studies with poorer methodological design and of different quality.

\section{Limitation of the review and implications for clinical practice}

Currently, it is not clear whether VBF might help with motivation and long-term adherence; hence, if it provides similar benefits as exercise, future studies should investigate the long-term adherence and motivation of VBF.

The use of VBF systems independently at home should be considered in future studies as commercial systems can be relatively inexpensive and do not require large spaces. Instead of participating in a formal exercise program that requires time and place, the Wii device is a suitable alternative method of training for the elderly, as they should engage in exercise programs to limit the negative effect of aging and a sedentary lifestyle. Several factors should be taken into account for future studies, such as individual age, balance performance, and cognitive abilities.

Future RCTs should focus upon trials with larger sample sizes, standardized protocols, and follow-up assessment. In addition, since most studies were of moderate quality, future studies should focus upon applying concealment of allocation of participants in their groups and reporting detailed information on blinding of the assessors.

A limitation within this SR is that only published studies were included, the grey literature was overlooked, which could indicate publication bias as most studies reported positive outcomes. The World Health Organization (WHO) defines elderly and old persons as any individual aged $\geq 65$ years; there are some arguments with regard to the age as according to the United Nations, they recognized people aged 60 years as old persons, in most developed countries the age of 60 or 65 years is equivalent to retirement age which is the beginning of old age. ${ }^{25}$ In the literature most of the time, the definition of old persons is linked to retirement age. The current study followed the definition set by WHO.

\section{Conclusion}

VBF was shown to have significant effect on balance across the included studies; however, the effect size was mainly small. The clinical relevance from the review of five RCTs suggests that engaging elderly people living in the community in VBF training is effective and could improve their balance ability. Nevertheless, the variation between studies in methodology, intervention protocol, and outcomes utilized made it difficult to inform a definitive statement regarding the potential application of VBF as a balance training for elderly. The overall quality of evidence in this SR is of moderate quality based upon The Grading of Recommendations, Assessment, Development, and Evaluations. ${ }^{26}$ The strength of recommendation was based upon Strength-of-Recommendation Taxonomy; ${ }^{27}$ the strength of recommendation in favor of VBF would be considered a level $\mathrm{B}$, which indicates that VBF is likely to be beneficial. 


\section{Disclosure}

The authors report no conflicts of interest in this work.

\section{References}

1. Barin K, Dodson E. Dizziness in the elderly. Otolaryngol Clin North Am. 2011;44(2):437-454.

2. Rubenstein L, Josephson K. The epidemiology of falls and syncope. Clin Geriatr Med. 2002;18(2):141-158.

3. Lin H, Bhattacharyya N. Balance disorders in the elderly: epidemiology and functional impact. Laryngoscope. 2012;122(8):1858-1861.

4. Roig M, Eng J, Road J, Reid W. Falls in patients with chronic obstructive pulmonary disease: a call for further research. Respir Med. 2009;103(9): $1257-1269$.

5. NICE Guidelines [CG161]. Falls: The Assessment and Prevention of Falls in Older People. London: National Institute for Clinical Excellence; 2013.

6. Huxham F, Goldie P, Patla A. Theoretical considerations in balance assessment. Aust J Physiother. 2001;47(2):89-100.

7. Frank L, Peter C. Understanding frailty. Postgrad Med J. 2007;83(975): $16-20$.

8. Ma CZ, Wan AH, Wong DW, Zheng YP, Lee WC. A vibrotactile and plantar force measurement-based biofeedback system: paving the way towards wearable balance-improving devices. Sensors. 2015;15(12): 31709-31722.

9. Goble D, Coxon J, Wenderoth N, Van Impe A, Swinnen S. Proprioceptive sensibility in the elderly: degeneration, functional consequences and plastic-adaptive processes. Neurosci Biobehav Rev. 2009; 33(3):271-278.

10. Laufer Y, Dar G, Kodesh E. Does a Wii-based exercise program enhance balance control of independently functioning older adults? A systematic review. Clin Interv Aging. 2014;9(1):1803-1813.

11. Rodrigues V, Sílvia R, Liliana L, Anna R. Effects of video game training on the musculoskeletal function of older adults: a systematic review and meta-analysis. Top Geriatr Rehabil. 2014;30(4):238-245.

12. Agnes Z, Martina M, Lorenzo C, Wiebren Z. Biofeedback for training balance and mobility tasks in older populations: a systematic review. J Neural Eng Rehabil. 2010;7(1):58. Available from: http://www.ncbi. nlm.nih.gov/pmc/articles/PMC3019192/. Accessed January 19, 2016.

13. Berg K, Wood-Dauphinee S, Williams J, Gayton D. Measuring balance in the elderly: preliminary development of an instrument. Physiother Can. 1989;41(6):304-311.
14. Podsiadlo D, Richardson S. The timed 'Up and Go': a test of basic functional mobility for frail elderly persons. J Am Geriatr Soc. 1991; 39(2):142-148.

15. Herd C, Meserve B. A systematic review of the effectiveness of manipulative therapy in treating lateral epicondylalgia. J Man Manip Ther. 2008;16(4):225-237.

16. Moseley A, Herbert R, Sherrington C, Maher C. Evidence for physiotherapy practice: a survey of the Physiotherapy Evidence Database (PEDro). Aust J Physiother. 2002;48(1):43-49.

17. Sullivan G, Feinn R. Using effect size - or why the $P$ value is not enough. J Grad Med Educ. 2012;4(3):279-282.

18. Rendon A, Lohman E, Thorpe D, Johnson E, Medina E, Bradley B. The effect of virtual reality gaming on dynamic balance in older adults. Age Ageing. 2012;41(4):549-552.

19. Jorgensen G, Uffe L, Carsten H, Bruno N, Per A. Efficacy of Nintendo Wii training on mechanical leg muscle function and postural balance in community dwelling older adults: a randomized controlled trial. J Gerontol. 2013;68(7):845-852.

20. Yoo H, EunJung C, Byoung L. The effects of augmented reality-based Otago exercise on balance, gait, and falls efficacy of elderly women. J Phys Ther Sci. 2013;25(7):797-801.

21. Cho G, Gak H, Hyung S. The effects of virtual reality-based balance training on balance of the elderly. J Phys Ther Sci. 2014;26(4):615-617.

22. Park E, Seong-Gil K, Chae-Woo L. The effects of virtual reality game exercise on balance and gait of the elderly. J Phys Ther Sci. 2015; 27(4):1157-1159.

23. Goble D, Cone L, Fling W. Using the Wii Fit as a tool for balance assessment and neurorehabilitation: the first half decade of wii-search. J Neuroeng Rehabil. 2014;11(1):12-19.

24. Chodzko-Zajko WJ, Proctor DN, Singh MAF, et al. American College of Sports Medicine position stand: exercise and physical activity for older adults. Med Sci Sports Exer. 2009;41(7):1510-1530.

25. Gorman M. Development and the rights of older people. In: Randel J, et al, editors. The Ageing and Development Report: Poverty, Independence and the World's Older People. London: Earthscan Publications Ltd.; 1999:3-21.

26. Goldet G, Howick J. Understanding GRADE: an introduction. $J$ Evid Based Med. 2013;6(1):50-54.

27. Ebell M, Siwek J, Weiss B, et al. Strength of recommendation taxonomy (SORT): a patient-centered approach to grading evidence in the medical literature. Am Fam Physician. 2004;69(3):548-556. 


\section{Supplementary material}

Table SI MEDLINE and EMBASE search strategy

\begin{tabular}{llll}
\hline I & Biofeedback, Psychology/ & 16 & posture.mp. \\
2 & biofeedback.mp. & 17 & Musculoskeletal Equilibrium.mp. \\
3 & bio-feedback.mp. & 18 & I2 or I3 or I4 or 15 or 16 or 17 \\
4 & Augmented feedback.mp. & 19 & Aged/ \\
5 & Feedback, Sensory/ & 20 & aged.mp. \\
6 & Visual feedback.mp. & 21 & Older people.mp. \\
7 & Video Games/ & 22 & Old people.mp. \\
8 & Computer games.mp. & 23 & Older adults.mp. \\
9 & Video Games.mp. & 24 & Older persons.mp. \\
10 & Virtual reality.mp. & 25 & Old persons.mp. \\
11 & I or 2 or 3 or 4 or 5 or 6 or 7 or 8 or 9 or 10 & 26 & Older subjects.mp. \\
12 & Postural Balance/ & 27 & Old subjects.mp. \\
13 & balance.mp. & 28 & elderly.mp. \\
14 & equilibrium.mp. & 29 & I9 or 20 or 21 or 22 or 23 or 24 or 25 or 26 or 27 or 28 \\
15 & Posture/ & 30 & II and I8 and 29 \\
\hline
\end{tabular}

Clinical Interventions in Aging

\section{Publish your work in this journal}

Clinical Interventions in Aging is an international, peer-reviewed journal focusing on evidence-based reports on the value or lack thereof of treatments intended to prevent or delay the onset of maladaptive correlates of aging in human beings. This journal is indexed on PubMed Central, MedLine,
Dovepress

CAS, Scopus and the Elsevier Bibliographic databases. The manuscript management system is completely online and includes a very quick and fair peer-review system, which is all easy to use. Visit http://www.dovepress. $\mathrm{com} /$ testimonials.php to read real quotes from published authors. 